\title{
Toxocara canis and the allergic process
}

\author{
Mauricio Grecco Zaia', Sandra Regina Pereira de Oliveiraㅁ, Cynthia Aparecida de Castro ${ }^{2}$, \\ Edson Garcia Soares ${ }^{3}$, Ana Afonso ${ }^{1,4,5}$, Luis Gustavo S Monnazzi ${ }^{6}$, Oscar Peitl Filho ${ }^{7}$, \\ Lúcia Helena Faccioli ${ }^{8}$, Fernanda de Freitas Anibal1/++
}

\begin{abstract}
1'Universidade Federal de São Carlos, Centro de Ciências Biológicas e da Saúde, Departamento de Morfologia e Patologia, Laboratório de Parasitologia, São Carlos, SP, Brasil ²Universidade Federal de São Carlos, Centro de Ciências Biológicas e da Saúde, Departamento de Ciências Fisiológicas, São Carlos, SP, Brasil ${ }^{3}$ Universidade de São Paulo, Faculdade de Medicina de Ribeirão Preto, Departamento de Patologia e Medicina Legal, Ribeirão Preto, SP, Brasil ${ }^{4}$ Universidade Nova de Lisboa, Instituto de Higiene e Medicina Tropical, Unidade de Parasitologia Médica, Lisboa, Portugal ${ }^{5}$ Universidade de São Paulo, Instituto de Química de São Carlos, Grupo de Bioanalítica, Microfabricação e Separações, São Carlos, SP, Brasil ${ }^{6}$ Centro Universitário de Araraquara, Centro de Ciências Biológicas e da Saúde, Araraquara, SP, Brasil ${ }^{7}$ Universidade Federal de São Carlos, Curso de Engenharia de Materiais, Departamento de Engenharia de Materiais, São Carlos, SP, Brasil ${ }^{8}$ Universidade de São Paulo, Faculdade de Ciências Farmacêuticas de Ribeirão Preto, Departamento de Análises Clínicas, Toxicológicas e Bromatológicas, Ribeirão Preto, SP, Brasil
\end{abstract}

The protective effect of infectious agents against allergic reactions has been thoroughly investigated. Current studies have demonstrated the ability of some helminths to modulate the immune response of infected hosts. The objective of the present study was to investigate the relationship between Toxocara canis infection and the development of an allergic response in mice immunised with ovalbumin (OVA). We determined the total and differential blood and bronchoalveolar lavage fluid cells using BALB/c mice as a model. To this end, the levels of interleukin (IL)-4, IL-5 and IL-10 and anti-OVA-IgE were measured using an ELISA. The inflammatory process in the lungs was observed using histology slides stained with haematoxylin and eosin. The results showed an increase in the total number of leukocytes and eosinophils in the blood of infected and immunised animals at 18 days after infection. We observed a slight lymphocytic inflammatory infiltrate in the portal space in all infected mice. Anti-OVA-IgE levels were detected in smaller proportions in the plasma of immunised and infected mice compared with mice that were only infected. Therefore, we concluded that $\mathrm{T}$. canis potentiates inflammation in the lungs in response to OVA, although anti-OVAIgE levels suggest a potential reduction of the inflammatory process through this mechanism.

Key words: toxocariasis - ELISA - eosinophils - IgE - lungs

Toxocara canis is an intestinal nematode that affects dogs. In humans, this geohelminth induces visceral larva migrans (VLM) syndrome, which is associated with severe eosinophilia, increased serum IgE and inflammation of the airways (Rogerio et al. 2003). Humans become infected after ingestion of the embryonated eggs, primarily in public parks and sandboxes that have been contaminated with animal faeces. The larvae are released into the intestinal walls and migrate to different organs, including the liver and lungs (Pinelli et al. 2007), causing fever, hepatosplenomegaly and respiratory dysfunction such as cough, wheezing and air flow obstruction (Qualizza et al. 2009). T. canis larvae induce a T-helper (Th)2 response, resulting in the secretion of interleukin (IL)-4 and the subsequent production of $\operatorname{IgE}$ and IL-5, as well as the differentiation and activation of eosinophils (Qualizza et al. 2009). Helminthic infections and allergic reactions have long been described as simi-

doi: 10.1590/0074-02760150051

Financial support: FAPESP

+ Corresponding author: ffanibal@ufscar.br

Received 6 February 2015

Accepted 10 July 2015 lar. The increase in the prevalence of allergic diseases/ reactions has been associated with a reduction of infections primarily in developed countries, according to the hygiene hypothesis (Moncayo \& Cooper 2006). The environment shows great influence in the hyper reactivity of the lungs and models the reactions in this organ (Wilson et al. 2005). There is robust evidence demonstrating immune suppression during helminthic infections (Medeiros Jr et al. 2003, Erb 2009). Epidemiological data have demonstrated that Schistosoma spp infections have great protective effects against allergic affections and Necator americanus protects against asthma (Okada et al. 2010). Thus, parasitic infections enhance IL-10 production, which, in turn, is inversely associated with allergic sensibility. A high IgE concentration serves as an indicator of the development of allergic disease in neonates and is an indicator of prognosis in adults with certain chronic allergic diseases (Sorensen \& Sakali 2006). T. canis has also been studied as a potential allergy suppressor. Although some studies have indicated that $T$. canis infection exacerbates allergic reactions (Buijs et al. 1997, Wohlleben et al. 2004, Pinelli et al. 2007), additional studies are needed to better understand and confirm these findings. In the present study, we established the relationship between T. canis infection and lung hyperreactivity in BALB/c mice immunised with ovalbumin (OVA). The number of leukocytes (polymorphonuclear and mononuclear cells and eosinophils) 
TABLE

Experimental design for immunisation and challenge with ovalbumin (OVA)

\begin{tabular}{lccc}
\hline $\begin{array}{l}\text { Experimental } \\
\text { group }\end{array}$ & $\begin{array}{c}\text { Toxocara canis } \\
\text { infection }\end{array}$ & $\begin{array}{c}\text { OVA } \\
\text { immunisation }\end{array}$ & $\begin{array}{c}\text { OVA } \\
\text { challenge }\end{array}$ \\
\hline Control & No & No & 12 th and 17th p.i. \\
OVA & No & 0 and 7th p.i. & 12 th and 17th p.i. \\
T. canis & Yes & No & 12 th and 17th .i. \\
OVA + T. canis & Yes & 0 and 7th p.i. & 12 th and 17th p.i.
\end{tabular}

p.i.: post-infection.

in the blood and bronchoalveolar lavage fluid (BALF) was counted and serum IL-4, IL-5, IL-10 and OVA-IgE levels were determined. Pulmonary inflammation was evaluated using histological sections stained with haematoxylin and eosin (H\&E). These new data will be of great importance to corroborate the relationship between $T$. canis and allergy and confirm previous results obtained in animal models for allergy using OVA.

\section{MATERIALS AND METHODS}

Animals - Female BALB/c specific pathogens free mice at six-eight weeks of age and weighing 15-20 g were obtained from the animal facilities of the School of Pharmaceutical Sciences of Ribeirão Preto, University of São Paulo, Brazil. These animals were maintained under standard laboratory conditions throughout the experimental period at the Laboratory of Parasitology, Department of Morphology and Pathology, Federal University of São Carlos (UFSCar), Brazil, with free access to water and food. This project was approved by the Ethical Committee on Animal Use of UFSCar (CEA 056/2011).

Mice infection with T. canis - T. canis eggs were obtained according to the method of Olson and Schutz (1963) with modifications, according to Faccioli et al. (1996). Briefly, pregnant female worms were recovered from infected dogs and the eggs were collected from the uterus of these worms. Subsequently, the eggs were washed and incubated at $37^{\circ} \mathrm{C}$ in $2 \%$ formalin to facilitate progression to the infectious stage. On day 0, 12 mice were infected through an intragastric route with $0.2 \mathrm{~mL}$ of saline containing 500 embryonated T. canis eggs.

Immunisation and challenge with OVA - Animal immunisation was performed on days 0 and 7 through the subcutaneous injection of $4 \mu \mathrm{g}$ of OVA and $1.6 \mathrm{mg}$ aluminium hydroxide in $0.4 \mathrm{~mL}$ saline. All animals were challenged twice through an intranasal route (at 12 and 17 days post-immunisation) with $10 \mu \mathrm{g}$ of OVA in 50 $\mu \mathrm{L}$ of saline, delivered into the nostrils. All assays were performed at $24 \mathrm{~h}$ after the second challenge [at 18 days post-infection (p.i.)] and six mice from each group were sacrificed. Two sets of experiments were performed under the same conditions (Russo et al. 2001). There were four groups per experiment: control (challenged with OVA at 12 and 17 days p.i.), OVA (immunised subcutaneously on days 0 and 7 and challenged with OVA at 12 and 17 days p.i.), T. canis (infected with T. canis on day 0 and challenged with OVA at 12 and 17 days p.i.) and OVA $+T$. canis (infected with $T$. canis on day 0 , immunised subcutaneously on days 0 and 7 and challenged with OVA at 12 and 17 days p.i.) (Table).

Extraction of fluids and cell counts - The mice were anaesthetised with sodium pentobarbitone $(30 \mathrm{mg} / \mathrm{kg}$ intravenous) and the peripheral blood (PB) samples were obtained through cardiac puncture. Absolute leukocyte counts were measured after counting in a Neubauer chamber. PB was collected using ethylenediamine tetraacetic acid (EDTA) as an anticoagulant. The absolute number of different leukocytes (mononuclear and polymorphonuclear cells and eosinophils) was obtained from differential counts on blood smears stained with Panótico Rápido LB (Laborclin Ltda, Brazil).

The cells in the peritoneal cavity (PC) were collected after injection with $3 \mathrm{~mL}$ of phosphate-buffered saline (PBS) containing $0.5 \%$ sodium citrate. To collect the BALF, a polyethylene cannula was introduced in the trachea and $1 \mathrm{~mL}$ of PBS/sodium citrate was injected. These procedures were repeated twice to obtain a greater number of cells. The total number of leukocytes in the PC and BALF was counted in a Neubauer chamber and the differential count was obtained from slides prepared using cytospin (SEROCITO mod. 2400; FANEM, Brazil) (1,000 rpm/3 min) and stained with Panótico Rápido LB.

Cytokines - Commercially available antibodies for the ELISA were used to measure the levels of IL-4, IL-5 and IL-10 in the plasma according to the manufacturer's instructions (BD OptEIA ${ }^{\mathrm{TM}}$; BD Biosciences).

$\operatorname{IgE}$ anti-OVA - ELISAs were performed according to the methods of Schnare et al. (2001) and (Rogerio et al. 2003) with modifications. Polystyrene microtitration plates (Greiner Bio-One) were coated with OVA, Chicken E19 Soma A-5253 (100 $\mu \mathrm{L} /$ well), at a concentration of $10 \mu \mathrm{g} / \mathrm{mL}$ in $0.1 \mathrm{M}$ carbonate-bicarbonate buffer (CBB), $\mathrm{pH} 9.6$, for $18 \mathrm{~h}$ at $4^{\circ} \mathrm{C}$. The plates were blocked with $10 \%$ skimmed milk in $1 \mathrm{X}$ PBS for $2 \mathrm{~h}$ at room temperature (RT) $(200 \mu \mathrm{L} /$ well $)$ and washed three times with PBS$\mathrm{T}$ (PBS $+0.1 \%$ Tween 20) using a microplate washer (Bras Serum, model BS II). The serum was diluted (1:1) in PBS and incubated for $2 \mathrm{~h}$ at room temperature (100 $\mu \mathrm{L} /$ well), followed by washing three times. Anti-mouse 
IgE monoclonal antibody (BD Biosciences) in $\mathrm{CBB}$ was added $(100 \mu \mathrm{L} /$ well) and incubated for $1 \mathrm{~h}$. After washing, biotinylated anti-mouse IgE monoclonal antibody in $1 \mathrm{X}$ PBS was added $(100 \mu \mathrm{L} /$ well $)$ and incubated for $1 \mathrm{~h}$. Streptavidin-horseradish peroxidase conjugate was added and incubated for $1 \mathrm{~h}(100 \mu \mathrm{L} /$ well). Subsequently, the reaction was developed for 20 min using a 3,3',5,5'-tetramethylbenzidine substrate $(50 \mu \mathrm{L} /$ well $)$ and terminated after the addition of $50 \mu \mathrm{L} 0.1 \mathrm{M}$ sulphuric acid to each well. The absorbance at $450 \mathrm{~nm}$ was measured using an automatic microplate reader (Tecan SLT Spectra).

Histology - The lungs were removed from the mice and immediately fixed in $10 \%$ formalin at 18 days after infection. The specimens were routinely processed, embedded in paraffin blocks and sectioned into $5 \mu \mathrm{m}$ thick sections followed by staining with H\&E for examination under light microscopy. The slides were photographed at 100X and 500X magnification using a Leica DMRX microscope equipped with a suitable camera.

Statistical analysis - Each experiment was performed twice and the data analysis was performed using one-way ANOVA, followed by Bonferroni's correction for multiple comparisons, using GraphPad Prism 5. Differences were considered significant at a $p$ value $<0.05$.

\section{RESULTS}

Leukocytes - The number of leukocytes in the blood of the OVA $+T$. canis group was significantly higher than that in the other experimental groups, except OVA $(p<0.05)$. In the BALF, no significant difference was observed (Fig. 1).

Mononuclear and polymorphonuclear cells - Analysis of the mononuclear cells present in the blood showed that OVA $+T$. canis was the only experimental group that displayed a significant increase in cell number compared with the other three experimental groups $(p<0.05)$. In the BALF, T. canis and OVA $+T$. canis experimental groups presented a significant increase in mononuclear cells compared with the OVA group $(\mathrm{p}<0.05)$. No significant relationship between the other groups was observed.

There was no statistically significant difference in the number of blood polymorphonuclear cells between the control, OVA and T. canis experimental groups ( $\mathrm{p}$ $>0.05$ ). However, the OVA $+T$. canis group showed an increase in the number of polymorphonuclear cells compared with the other experimental groups. In the BALF, the number of polymorphonuclear cells in all experimental groups was significantly higher compared with the control group $(\mathrm{p}<0.05)$. Although the values for $T$. canis were higher than in the control group, these values were significantly lower compared with the OVA and $\mathrm{OVA}+$ T. canis experimental groups (Fig. 2).

Eosinophils - In the blood, we observed an increase in the eosinophil number in all groups compared with the control group. Eosinophils cell numbers obtained in T. canis experimental group was lower than in the OVA group. Animals also presented an increase in eosinophils in the OVA $+T$. canis group compared with the OVA and T. canis groups. The results obtained from the BALF revealed that OVA and OVA $+T$. canis groups presented a

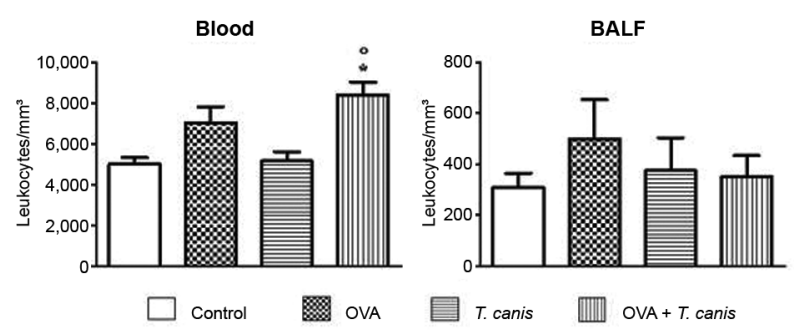

Fig. 1: total number of leukocytes in blood and bronchoalveolar lavage fluid (BALF). On the 18th day after Toxocara canis infection, the animals were sacrificed and the different biological materials were obtained and analysed. The data represent the mean \pm standard deviation ( $\mathrm{n}=6$ animals) of two independent experiments. Significant compared to control $(*)$ and T. canis $\left(^{\circ}\right)$. Differences were considered significant when $\mathrm{p}<0.05$. OVA: ovalbumin.
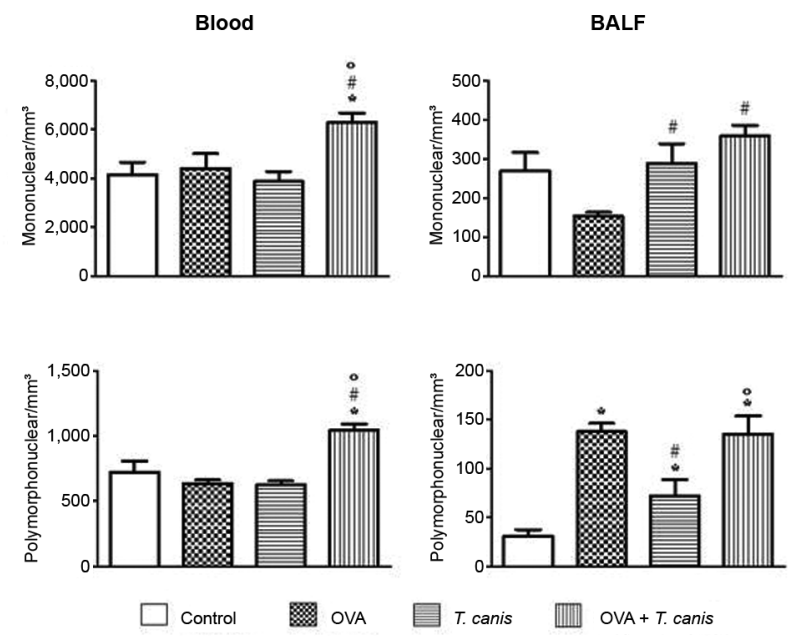

Fig. 2: total number of mononuclear and polymorphonuclear in blood and bronchoalveolar lavage fluid (BALF). On the 18th day after Toxocara canis infection the animals were sacrificed and the different biological materials were obtained and analysed. The data represent the mean \pm standard deviation ( $\mathrm{n}=6$ animals) of two independent experiments. Significant compared to control (*), ovalbumin (OVA) (\#) and T. canis $\left({ }^{\circ}\right)$. Differences were considered significant when $\mathrm{p}<0.05$.

significant increase in eosinophil numbers compared with the values obtained in the control and T. canis groups ( $\mathrm{p}$ $<0.05$ ). Although not significant, a slight reduction in the eosinophil number was observed in OVA $+T$. canis group compared with the OVA group in the BALF $(p>0.05)$. These results clearly suggest that the primary inducer of eosinophilia in this model was OVA, as the OVA group presented a higher number of these cells compared with the T. canis and OVA + T. canis groups (Fig. 3).

Cells from the $P C$ - The mononuclear and polymorphonuclear cells and PC eosinophils were obtained and counted. No significant differences were observed among the experimental groups, regardless of cell type. 


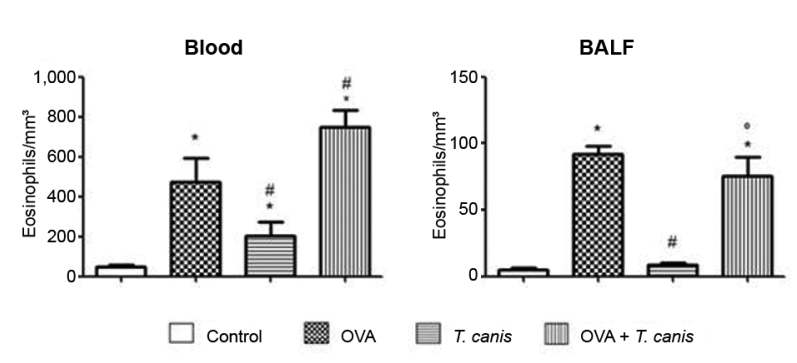

Fig. 3: total number of eosinophils in blood and bronchoalveolar lavage fluid (BALF). On the 18th day after Toxocara canis infection the animals were sacrificed and the different biological materials were obtained and analysed. The data represent the \pm standard deviation (n $=6$ animals) of two independent experiments. Significant compared to control (*), ovalbumin (OVA) (\#) and T. canis $\left({ }^{\circ}\right)$. Differences were considered significant when $\mathrm{p}<0.05$.

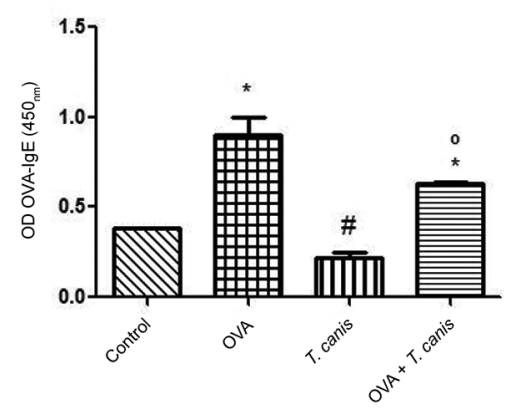

Fig. 4: ovalbumin (OVA)-IgE measure. Polystyrene microtitration plates (Greiner Bio-One) were coated with OVA, Chicken E19 Soma A-5253 $(100 \mu \mathrm{L} / w e l l)$, at a concentration of $10 \mu \mathrm{g} / \mathrm{mL}$ and an ELISA was realised to measure OVA-IgE in plasma of BALB/c mice. The results are shown as optical density (OD) OVA-IgE. Data represent triplicates of pooled plasma obtained 18 days after infection. Significant compared to control $(*)$, OVA (\#) and Toxocara canis $\left({ }^{\circ}\right)$. Differences were considered significant when $\mathrm{p}<0.05$.

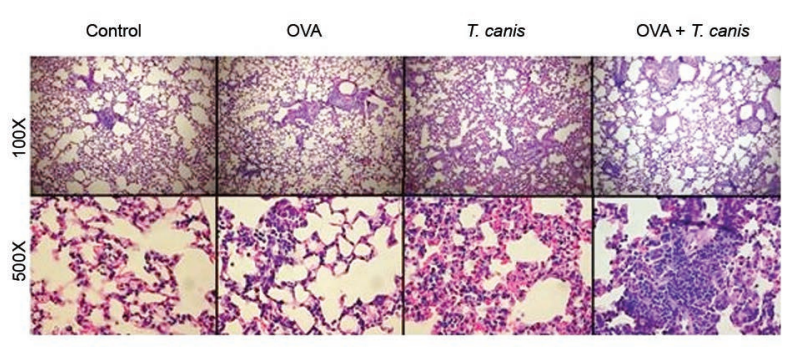

Fig. 5: lung histology stained with haematoxylin and eosin. Control, ovalbumin (OVA), Toxocara canis and OVA + T. canis groups are presented with 100X and 500X magnification.

OVA-IgE - The data obtained after measuring the OVA-IgE concentration showed that the animals infected with $T$. canis presented a decrease in the levels of OVA-IgE compared with the uninfected groups. The OVA $+T$. canis group showed a 1.4-fold decrease in the levels of OVA-IgE compared with the OVA group. In addition, the T. canis group also showed a 1.7-fold decrease compared with the control group (Fig. 4).

IL levels - The levels of IL-4, IL-5 and IL-10 were measured in mice plasma from all experimental groups using an ELISA. No changes were observed among the levels of measured ILs from all groups (data not shown).

Histology - To determine whether the inhibition of the inflammatory response and down-modulation of pathology resulted from infection, lung histological sections were compared between the OVA and OVA $+T$. canis experimental groups. The lung histological sections from the OVA experimental group presented peribronchial inflammation, predominantly lymphocyte in nature, with vascular congestion. Neutrophils and some eosinophils remained present throughout the lung parenchyma. Animals from OVA + T. canis experimental group displayed a more severe inflammatory peribronchial and intraseptal lymphocytic process. The predominance of lymphocytes and neutrophils and the widening of the alveolar septa were observed. The control group presented preserved cellular structures with rare inflammatory sites, presenting neutrophils and lymphocytes and occasional foci of intraalveolar haemorrhage. The T. canis group presented a much larger vascular congestion compared with the control and OVA experimental groups. Dense septal lengthening inflammatory infiltrate predominantly comprising lymphocytes, plasma cells and a few neutrophils were observed (Fig. 5).

\section{DISCUSSION}

Previous studies have demonstrated that helminthic infections might modulate a Th2 response in allergic patients (Sorensen \& Sakali 2006, Ponte et al. 2007, Okada 2010). In the present study, OVA was used as an allergy model to determine whether $T$. canis infection modulates and decreases the allergic inflammation response stimulated through OVA in BALB/c mice. Analyses of the cells in the blood and BALF showed interesting results. A comparison of the data obtained from the OVA and OVA $+T$. canis experimental groups showed an increase in all cell types in the infected group. The increase in the eosinophil numbers in the T. canis group might reflect the increased stimulation induced through secretion/excretion antigens from T. canis larvae. Toxocara sp. larvae secrete antigens with allergenic properties, suggesting that this parasite may generate high levels of total IgE (Roldán 2010). Some Toxocara sp. antigens induce a Th2 response with IL-4 production and subsequent differentiation of B-lymphocytes into plasma cells secreting IgE antibodies and IL-5 for the differentiation and activation of eosinophils (Qualizza et al. 2009). We hypothesised that the eosinophils present in the BALF primarily reflect OVA, because mice from the $T$. canis experimental group did not present this cell type. This result suggests that infection with $T$. canis stimulates the influx of eosinophils into $\mathrm{PB}$ in $\mathrm{BALB} / \mathrm{C}$ mice, but exerts no influence in the BALF. Mononuclear cells were predominantly observed in the blood from mice of the OVA $+T$. canis experimental group, indi- 
cating that when synergistically stimulated (OVA and $T$. canis infection), the function of lymphocytes is primarily induced, reflecting an increase in reactivity and the release of antigens. In addition, this group also showed the highest influx of leukocytes in BALF. We observed a significant increase on polymorphonuclear cell in the blood of OVA $+T$. canis experimental group compared with other experimental groups. In the BALF, this increase was observed in all groups compared with the control. OVA and OVA $+T$. canis experimental groups presented a higher number of polymorphonuclear compared with $T$. canis experimental group, suggesting that both $T$. canis larvae and particularly OVA stimulate the influx of polymorphonuclear cells into this site.

No differences in the levels of IL-4, IL-5 and IL-10 were observed (data not shown). Thus, we concluded that infection with both OVA and T. canis similarly stimulated the production of these cytokines and when animals were treated with both stimuli (OVA and $T . c a$ nis infection), the response pattern did not significantly change in this experimental design. Pinelli et al. (2005) showed that IL-4 and IL-10 levels in the BALF did not change during $T$. canis experimental infection, consistent with the results of the present study. Perhaps these results would have been different if the cytokine levels were evaluated through reverse transcription-polymerase chain reaction (RT-PCR). Post-transcriptional modifications might occur on mRNA, thereby compromising protein synthesis. ELISA results are not always consistent with RT-PCR data, although ELISA results are more conclusive. An interesting finding in the present study was obtained from the analysis of OVA-IgE. We observed a 1.4-fold decrease in OVA-IgE levels in OVA $+T$. canis mice compared with the OVA group. In the T. canis group, a 1.7-fold decrease was observed compared with the control group. This result strongly suggested that $T$. canis infection induced a decrease in OVA-IgE production and might reduce the release of anti-specific allergen (OVA). However, it was not possible to identify a decrease in inflammation in general, likely reflecting the increase in total $\mathrm{IgE}$ and the anti-soluble $\operatorname{IgE}$ antigens of $T$. canis. The results of the present study demonstrated that $T$. canis infection leads to damage in the lung epithelium, with peribronchial inflammation, eosinophil influx and widening of the alveolar septa. Most of the histological findings obtained from the lungs were consistent with previous data showing alterations in lung tissue associated with $T$. canis infection (Pinelli et al. 2006). We proposed that much of the damage reported in the present study is associated with T. canis infection, indicating that infection exacerbates the symptoms and the damage of the lung tissue, even under these experimental conditions. Previous studies with Heligmosomoides polygyrus have shown that parasites might suppress inflammation in allergic airways induced through OVA. Moreover, in models of chronic infection with Schistosoma mansoni, allergic reactions induced through OVA promote a decrease in pulmonary eosinophilia (van Riet et al. 2007). Inconsistencies among studies with helminths might reflect several fac- tors: age, genetics and helminth species (van Riet et al. 2007). Other studies have demonstrated that the type of infection might interfere with the response pattern. The results of a population study in Ethiopia showed that an intense infection with Ascaris sp. might contribute to the decreased wheezing in children (Dagoye et al. 2003). In addition, chronic helminthic infection is an important factor in the suppression of allergic inflammation (Moncayo \& Cooper 2006). However, the infection with T. canis was not associated with an improvement in chronic OVA-induced allergic manifestation (Pinelli et al. 2005). The results of the present study strongly suggested that acute infection is also unable to improve inflammation, even using a high parasite load and with a decrease in OVA-IgE. Differences in the results reported in previous studies conducted with $T$. canis might reflect the use of variant parasite specimens obtained in various parts of the world. Differences and similarities have been observed in Toxocara sp. (Chen et al. 2012), suggesting that further investigation of the strains used in the present study is needed. Thus, the characterisation of the strains/ lines of Toxocara might be useful for understanding this disease and in studies using the VLM model.

Several studies have suggested that some helminthic infections show therapeutic potential against immunopathology diseases. The results of the present study demonstrated that $T$. canis infection exacerbated experimental airway allergic inflammation in an acute infection model (18 days), which is consistent with previous data from human epidemiological studies and other animal models.

\section{REFERENCES}

Buijs J, Borsboom G, Renting M, Hilgersom WJA, van Wieringen JC, Jansen G, Neijens J 1997. Relationship between allergic manifestations and Toxocara seropositivity: a cross-sectional study among elementary school children. Eur Respir J 10: 1467-1475.

Chen J, Zhou DH, Nisbet AJ, Xu MJ, Huang SY, Li MW, Wang CR, Zhu XQ 2012. Advances in molecular identification, taxonomy, genetic variation and diagnosis of Toxocara spp. Infect Genet Evol 12: 1344-1348.

Dagoye D, Bekele Z, Woldemichael K, Nida H, Yimam M, Hall A, Venn AJ, Britton JR, Hubbard R, Lewis SA 2003. Wheezing, allergy and parasite infection in children in urban and rural Ethiopia. Am J Respir Crit Care Med 167: 1369-1373.

Erb KJ 2009. Can helminths or helminth-derived products be used in humans to prevent or treat allergic diseases? Trends Immunol 30: $75-82$.

Faccioli LH, Mokwa VF, Silva CL, Rocha GM, Araújo JI, Nahori MA, Vargaftig BB 1996. IL-5 drives eosinophils from bone marrow to blood and tissues in a visceral larva migrans syndrome. Mediators Inflamm 5: 24-31.

Medeiros Jr M, Figueiredo JP, Almeida MC, Matos MA, Araújo MI, Cruz AA, Atta AM, Rego MAV, Jesus AR, Taketomi EA, Carvalho EM 2003. Schistosoma mansoni infection is associated with a reduced course of asthma. J Allergy Clin Immunol 111: 947-951.

Moncayo AL, Cooper PJ 2006. Geohelminth infections: impact on allergic diseases. Int J Biochem Cell Biol 38: 1031-1035.

Okada H, Kuhn C, Feillet H, Bach J-F 2010. The "hygiene hypothesis" for autoimmune and allergic diseases: an update. Clin Exp Immunol 160: 1-9. 
Olson LJ, Schutz CV 1963. Nematode induced hypersensibility reactions in guinea pig: onset of eosinophilia and positive SchultzDale reactions following graded infection with Toxocara canis. Ann N Y Acad Sci 113: 440-455.

Pinelli E, Brandes S, Dormans J, Fonville M, Hamilton CM, van der Giessen J 2006. Toxocara canis: effect of inoculum size on pulmonary pathology and cytokine expression in BALB/c mice. Exp Parasitol 115: 76-82.

Pinelli E, Brandes S, Dormans J, Gremmer E, van Loveren H 2007. Infection with the roundworm Toxocara canis leads to exacerbation of experimental allergic airway inflammation. Clin Exp Allergy 38: 649-658.

Pinelli E, Withagen C, Fonville M, Verlaan A, Dormans J, van Loveren H, Nicoll G, Maizels RM, van der Giessens J 2005. Persistent airway hyper-responsiveness and inflammation in Toxocara canis infected BALB/c mice. Clin Exp Allergy 35: 826-832.

Ponte EV, Rizzo JA, Cruz AA 2007. Inter-relação entre asma, atopia e infecções helmínticas. J Bras Pneumol 33: 335-342.

Qualizza R, Megali R, Incorvaia C 2009. Toxocariasis resulting in seeming allergy. Iran J Allergy Asthma Immunol 8: 161-164.

Rogerio AP, Sá-Nunes A, Albuquerque DA, Anibal FF, Medeiros AI, Machado ER, Souza AO, Prado Jr JC, Faccioli LH 2003. Lafoen- sia pacari extract inhibits IL-5 production in toxocariasis. Parasite Immunol 25: 393-400.

Roldán WH 2010. Diagnóstico de la toxocarosis humana. Rev Peru Med Exp Salud Publica 27: 613-620.

Russo M, Nahori MA, Lefort J, Gomes E, Keller AC, Rodriguez D, Ribeiro OG, Adriouch S, Gallois V, de Faria AM, Vargaftig BB 2001. Suppression of asthma-like responses in different mouse strains by oral tolerance. Am J Respir Cell Mol Biol 24: 518-526.

Schnare M, Barton GM, Holt AC, Takeda K, Akira S, Medzhitov R 2001. Toll-like receptors control activation of adaptive immune responses. Nat Immunol 2: 947-950.

Sorensen RU, Sakali P 2006. Does parasitic infection protect against allergy? J Pediatr (Rio J) 82: 241-242.

van Riet E, Hartgers FC, Yazdanbakshs M 2007. Chronic helminth infections induce immunomodulation: consequences and mechanisms. Immunobiology 212: 475-490.

Wilson MS, Taylor MD, Balic A, Finney CAM, Lamb JR, Maizels RM 2005. Suppression of allergic airway inflammation by helminth-induced regulatory T-cells. J Exp Med 202: 1199-1212.

Wohlleben G, Trujillo C, Müller J, Ritze Y, Grunewald S, Tatsch U, Erb KJ 2004. Helminth infection modulates the development of allergen-induced airway inflammation. Int Immunol 16: 585-596. 